DOI 10.7603/s40956-016-0008-y

Moroccan J. Pure and Appl. Anal.(MJPAA)

RESEARCH ARTICLE

Volume 2(2), 2016, Pages 91-106

ISSN: $2351-8227$

\title{
Inclusion properties of Generalized Integral Transform using Duality Techniques
}

\author{
Satwanti Devi ${ }^{a}$ And A. Swaminathan ${ }^{b}$
}

Abstract. Let $\mathcal{W}_{\beta}^{\delta}(\alpha, \gamma)$ be the class of normalized analytic functions $f$ defined in the region $|z|<1$ and satisfying

$$
\begin{array}{r}
\operatorname{Re} e^{i \phi}\left((1-\alpha+2 \gamma)(f / z)^{\delta}+\left(\alpha-3 \gamma+\gamma\left[(1-1 / \delta)\left(z f^{\prime} / f\right)+1 / \delta\left(1+z f^{\prime \prime} / f^{\prime}\right)\right]\right)\right. \\
\left.(f / z)^{\delta}\left(z f^{\prime} / f\right)-\beta\right)>0,
\end{array}
$$

with the conditions $\alpha \geq 0, \beta<1, \gamma \geq 0, \delta>0$ and $\phi \in \mathbb{R}$. For a non-negative and realvalued integrable function $\lambda(t)$ with $\int_{0}^{1} \lambda(t) d t=1$, the generalized non-linear integral transform is defined as

$$
V_{\lambda}^{\delta}(f)(z)=\left(\int_{0}^{1} \lambda(t)(f(t z) / t)^{\delta} d t\right)^{1 / \delta}
$$

The main aim of the present work is to find conditions on the related parameters such that $V_{\lambda}^{\delta}(f)(z) \in \mathcal{W}_{\beta_{1}}^{\delta_{1}}\left(\alpha_{1}, \gamma_{1}\right)$, whenever $f \in \mathcal{W}_{\beta_{2}}^{\delta_{2}}\left(\alpha_{2}, \gamma_{2}\right)$. Further, several interesting applications for specific choices of $\lambda(t)$ are discussed.

2010 Mathematics Subject Classification. 30C45, 30C55, 32C15, 32C37, 33C20.

Key words and phrases. Integral Transforms, Analytic functions, Hypergeometric functions, Convolution, Duality techniques.

Received February 15, 2016 - Accepted June 16, 2016.

(C)The Author(s) 2016. This article is published with open access by Sidi Mohamed Ben Abdallah University

${ }^{a}$ Department of Applied Sciences The NorthCap University, Sector 23-A Gurgaon, Haryana, India. e-mail: ssatwanti@gmail.com, satwantidevi@ncuindia.edu

${ }^{b}$ Department of Mathematics Indian Institute of Technology, Roorkee, Uttarkhand, India. e-mail:swamifma@iitr.ac.in, mathswami@gmail.com 


\section{Introduction}

Let $\mathcal{A}$ be the class of all normalized and analytic functions $f$ defined in the open unit disk $\mathbb{D}=\{z \in \mathbb{C}:|z|<1\}$ that have the Taylor series representation

$$
f(z)=z+\sum_{n=2}^{\infty} a_{n} z^{n} .
$$

Let $\lambda(t):[0,1] \rightarrow \mathbb{R}$ be a non-negative integrable function which satisfies the condition $\int_{0}^{1} \lambda(t) d t=1$. For $f \in \mathcal{A}$, consider the generalized integral transform defined by

$$
F_{\delta}(f)(z):=V_{\lambda}^{\delta}(f)(z)=\left(\int_{0}^{1} \lambda(t)\left(\frac{f(t z)}{t}\right)^{\delta} d t\right)^{1 / \delta}, \quad \delta>0 \quad \text { and } \quad z \in \mathbb{D} .
$$

The power appearing in (1) and elsewhere in this manuscript are meant as principal values. We are interested in the following problem.

Problem 1.1. Find a class of admissible functions $f \in \mathcal{A}$ that are carried by the integral operator defined by (1) to a class of analytic functions.

Consideration of such problem for various type of non-linear integral operators exist in the literature. For example, the integral operator

$$
I_{g, c, \alpha}[f(z)]=\left(\frac{c+\alpha}{g^{c}(z)} \int_{0}^{z} f^{\alpha}(t) g^{c-1}(t) g^{\prime}(t) d t\right)^{1 / \alpha}
$$

where $g \in \mathcal{A}, g(0)=0, g^{\prime}(0) \neq 0$ and $g(z) \neq 0$ for $z \in \mathbb{D} \backslash\{0\}$, was considered by $\mathrm{T}$. Bulboacă in $[6$, P.58] for analyzing various inclusion properties involving interesting classes of analytic functions. Choosing $g(z)=z$ gives

$$
I_{c, \alpha}[f(z)]=\left(\frac{c+\alpha}{z^{c}} \int_{0}^{z} f^{\alpha}(t) t^{c-1} d t\right)^{1 / \alpha} .
$$

This operator has a rich literature and was considered among several authors by S. S.Miller and P.T. Mocanu [20, P.319] (see also [19, P.228]) for studying various inclusion properties. Even though particular values of the operator given by (2) may be related to the operator (1) under some restrictions, these two operators are entirely different. But the existing literature related to the operator (2) motivate us to consider suitable classes of analytic functions that can be studied with reference to the operator given by (1). For this purpose, we define the class $\mathcal{W}_{\beta}^{\delta}(\alpha, \gamma)$ in the following way.

$$
\begin{array}{r}
\mathcal{W}_{\beta}^{\delta}(\alpha, \gamma):=\left\{f \in \mathcal{A}: \operatorname{Re} e^{i \phi}\left((1-\alpha+2 \gamma)\left(\frac{f}{z}\right)^{\delta}+\left(\alpha-3 \gamma+\gamma\left[\left(1-\frac{1}{\delta}\right)\left(\frac{z f^{\prime}}{f}\right)+\frac{1}{\delta}\left(1+\frac{z f^{\prime \prime}}{f^{\prime}}\right)\right]\right)\right.\right. \\
\left.\left.\left(\frac{f}{z}\right)^{\delta}\left(\frac{z f^{\prime}}{f}\right)-\beta\right)>0, z \in \mathbb{D}, \phi \in \mathbb{R}\right\} .
\end{array}
$$

Note that, for the particular case $\mathcal{W}_{\beta}^{\delta}(\alpha, 0) \equiv P_{\alpha}(\delta, \beta)$, the operator (1) was examined by R. Aghalary et al. [1] using duality techniques. Further, for the case $\delta=1$, this operator (1) reduces to the one introduced by R. Fournier and S. Ruscheweyh [16], 
that contains some of the well-known operator such as Bernardi, Komatu and Hohlov as its special cases for particular choices of $\lambda(t)$, which has been extensively studied by various authors (for details see $[2,3,4,9,10,11]$ and references therein).

It is also interesting to note that results related to other particular cases namely, $\mathcal{W}_{\beta}^{1}(\alpha, \gamma) \equiv \mathcal{W}_{\beta}(\alpha, \gamma)$, considered by R.M. Ali et al [2] and $\mathcal{W}_{\beta}^{\delta}(\alpha+\delta+\delta \alpha, \delta \alpha) \equiv R_{\alpha}(\delta, \beta)$ exist in the literature. Note that $F:=z(f / z)^{\delta} \in R_{\alpha}(\delta, \beta) \Longleftrightarrow z F^{\prime}(z) \in P_{\alpha}(\delta, \beta)$. Hence the class $R_{\alpha}(\delta, \beta)$ is closely related to the class $P_{\alpha}(\delta, \beta)$. Inclusion properties of $V_{\lambda}^{\delta}(f)(z) \in \mathcal{W}_{\beta}^{\delta}(\alpha, \gamma)$ for subclasses of analytic functions that have geometrical meaning were considered by the authors of this work in $[12,13]$. Since it is challenging to solve Problem 1.1 completely, in this manuscript the following particular cases are addressed.

Problem 1.2. For given $\xi<1$, obtain the sharp bounds for $\beta$ such that

(i) $V_{\lambda}^{\delta}(f)(z) \in \mathcal{W}_{\xi}^{\delta}(1,0)$ whenever $f(z) \in \mathcal{W}_{\beta}^{\delta}(\alpha, \gamma)$ and

(ii) $V_{\lambda}^{\delta}(f)(z) \in \mathcal{W}_{\xi}^{\delta}(\alpha, \gamma)$ whenever $f(z) \in \mathcal{W}_{\beta}^{\delta}(\alpha, \gamma)$.

Main results related to Problem 1.2 and its consequences are given in Section 2 whereas the required proofs are given in Section 3 separately to provide the readers a collective view of the results.

\section{Main results and their consequences}

The following Lemma is used in obtaining the main results.

Lemma 2.1. [22] Let $\beta_{1}, \beta_{2}<1$ and $\phi \in \mathbb{R}$. Then for the analytic functions $p$ and $q$ defined in the region $\mathbb{D}$ with $p(0)=1=q(0)$, along with the conditions $\operatorname{Re} e^{i \phi}(p(z)-$ $\left.\beta_{1}\right)>0$ and $\operatorname{Re}\left(q(z)-\beta_{2}\right)>0$ implies $\operatorname{Re} e^{i \phi}((p(z) * q(z))-\beta)>0$, where $(1-\beta)=$ $2\left(1-\beta_{1}\right)\left(1-\beta_{2}\right)$.

Here $*$ denotes the convolution or Hadamard product of two normalized analytic functions $f_{1}(z)=z+\sum_{n=2}^{\infty} a_{n} z^{n}$ and $f_{2}(z)=z+\sum_{n=2}^{\infty} b_{n} z^{n}$, defined in $\mathbb{D}$, given by

$$
\left(f_{1} * f_{2}\right)(z)=z+\sum_{n=2}^{\infty} a_{n} b_{n} z^{n} .
$$

The parameters $\mu, \nu \geq 0$ introduced in [2] are used for further analysis that are defined by the following relations

$$
\mu \nu=\gamma \quad \text { and } \quad \mu+\nu=\alpha-\gamma
$$

Clearly (3) leads to two cases.

(i) $\gamma=0 \Longrightarrow \mu=0, \nu=\alpha \geq 0$.

(ii) $\gamma>0 \Longrightarrow \mu>0, \nu>0$.

Following theorem addresses the first question of Problem 1.2 and the proof of the same is given in Section 3. 
Theorem 2.1. Let $\gamma \geq 0(\mu \geq 0, \nu \geq 0)$ and $\delta>0$. Further let $\xi<1$ and $\beta<1$ be defined by the relation

$$
\beta=\left\{\begin{array}{cc}
1-\frac{1-\xi}{2}\left(1-\int_{0}^{1} \lambda(t)\left[\frac{1}{\nu} \int_{0}^{1} \frac{d s}{1+t s^{\mu / \delta}}+\left(1-\frac{1}{\nu}\right) \int_{0}^{1} \int_{0}^{1} \frac{d r d s}{1+t r^{\nu / \delta} s^{\mu / \delta}}\right] d t\right)^{-1} & \gamma>0, \\
1-\frac{1-\xi}{2}\left(1-\int_{0}^{1} \lambda(t)\left[\frac{1}{\alpha(1+t)}+\left(1-\frac{1}{\alpha}\right) \int_{0}^{1} \frac{d r}{1+t r^{\alpha / \delta}}\right] d t\right)^{-1} & \gamma=0 .
\end{array}\right.
$$

Then for $f(z) \in \mathcal{W}_{\beta}^{\delta}(\alpha, \gamma)$, the function $V_{\lambda}^{\delta}(f)(z) \in \mathcal{W}_{\xi}^{\delta}(1,0)$. The value of $\beta$ is sharp.

Remark 2.1. (1) For $\gamma=0$, Theorem 2.1 reduces to [1, Theorem 2.1].

(2) For $\delta=1$, Theorem 2.1 gives the result of [23, Theorem 2.1].

The integral operator (1) defined by the weight function

$$
\lambda(t)=(1+c) t^{c}, \quad c>-1,
$$

is known as generalized Bernardi operator denoted by $\left(\mathcal{B}_{c}^{\delta}\right)$. This operator is the particular case of the generalized integral operators, considered in the work of $\mathrm{R}$. Aghalary et al. [1] that follows in the sequel. The operator corresponding to the value $\delta=1$, was introduced by S. D. Bernardi [7]. Now, using the integral operator $\mathcal{B}_{c}^{\delta}$, the following corollary is stated as under.

Corollary 2.1. Let $\gamma \geq 0(\mu \geq 0, \nu \geq 0), \delta>0$ and $c>-1$. Further let $\xi<1$ and $\beta<1$ be defined by the relation

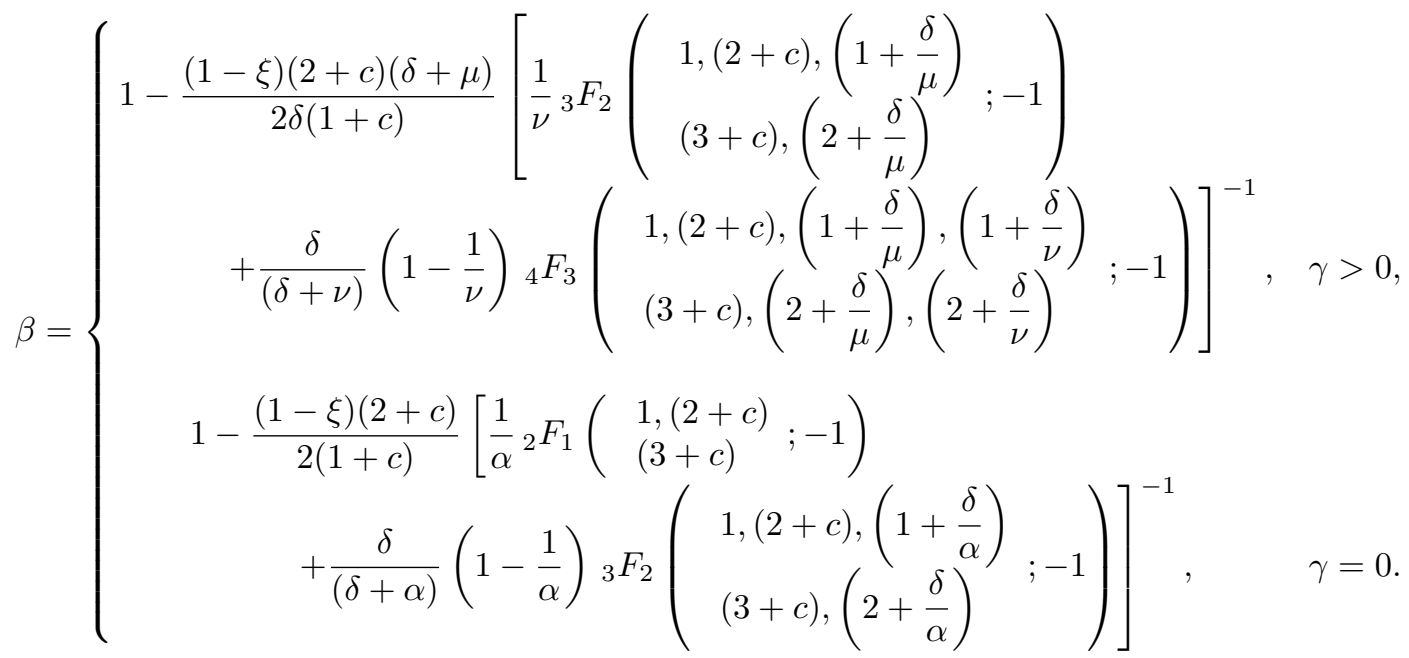

Then for $f(z) \in \mathcal{W}_{\beta}^{\delta}(\alpha, \gamma)$, the function

$$
\mathcal{B}_{c}^{\delta}(f)(z)=\left(\frac{(1+c)}{z^{c-\delta+1}} \int_{0}^{z} \omega^{c-\delta}(f(\omega))^{\delta} d \omega\right)^{1 / \delta},
$$

belongs to $\mathcal{W}_{\xi}^{\delta}(1,0)$. The value of $\beta$ is sharp. 
Here ${ }_{p} F_{q}\left(c_{1}, \ldots, c_{p} ; d_{1}, \ldots, d_{q} ; z\right)$ or ${ }_{p} F_{q}$ denotes the generalized hypergeometric function given by

$$
{ }_{p} F_{q}\left(\begin{array}{l}
c_{1}, \ldots, c_{p} \\
d_{1}, \ldots, d_{q}
\end{array} ; z\right)=\sum_{n=0}^{\infty} \frac{\left(c_{1}\right)_{n} \ldots,\left(c_{p}\right)_{n}}{\left(d_{1}\right)_{n} \ldots,\left(d_{q}\right)_{n} n !} z^{n}, \quad z \in \mathbb{D}
$$

where $c_{i}(i=0,1, \ldots, p)$ and $d_{j}(j=0,1, \ldots, q)$ are the complex parameters with $d_{j} \neq$ $0,-1, \ldots$ and $p \leq q+1$. In particular, ${ }_{2} F_{1}$ is the well-known Gaussian hypergeometric function. For any natural number $n$, the Pochhammer symbol or shifted factorial $(\varepsilon)_{n}$ is defined as $(\varepsilon)_{0}=1$ and $(\varepsilon)_{n}=\varepsilon(\varepsilon+1)_{n-1}$. The generalized hypergeometric series ${ }_{p} F_{q}$ defined in (6), converges absolutely for all $z$ in $|z|<\infty$ if $p<q+1$, and for $z \in \mathbb{D}$ if $p=q+1$.

The following result gives conditions such that $V_{\lambda}^{\delta}(f)(z) \in \mathcal{W}_{\xi}^{\delta}(\alpha, \gamma)$ whenever $f(z) \in$ $\mathcal{W}_{\beta}^{\delta}(\alpha, \gamma)$ which addresses the second part of Problem 1.2.

Theorem 2.2. Let $\gamma \geq 0(\mu \geq 0, \nu \geq 0)$ and $\delta>0$. Further let $\xi<1$ and $\beta<1$ be defined by the relation

$$
\frac{\beta}{(1-\beta)}=-\int_{0}^{1} \lambda(t) \frac{\left(1-\frac{1+\xi}{1-\xi} t\right)}{1+t} d t
$$

Then for $f(z) \in \mathcal{W}_{\beta}^{\delta}(\alpha, \gamma)$, the function $V_{\lambda}^{\delta}(f)(z) \in \mathcal{W}_{\xi}^{\delta}(\alpha, \gamma)$. The value of $\beta$ is sharp.

It is interesting to note that Theorem 2.2 cannot be reduced to Theorem 2.1 for particular values of the parameters. However, for particular values of the parameters given in Theorem 2.2 several results exist in the literature and few of them are listed below.

Remark 2.2. (1) For $\gamma=0, \delta=1, \alpha=1$ Theorem 2.2 reduces to [16, Theorem 2].

(2) For $\gamma=0$ and $\delta=1$, Theorem 2.2 gives the result of [17, Theorem 2.6].

(3) For $\gamma=0$, Theorem 2.2 reduces to [1, Theorem 2.2].

(4) For $\delta=1$, Theorem 2.2 gives the result of [23, Theorem 2.3].

On setting $\lambda(t)$ given in (5), Theorem 2.2 will lead to the following result.

Corollary 2.2. Let $\gamma \geq 0(\mu \geq 0, \nu \geq 0), \delta>0$ and $c>-1$. Further let $\xi<1$ and $\beta<1$ be defined by the relation

$$
\beta=\frac{2(1+c){ }_{2} F_{1}(1,2+c ; 3+c ;-1)-(2+c)(1-\xi)}{2(1+c){ }_{2} F_{1}(1,2+c ; 3+c ;-1)} .
$$

Then for $f(z) \in \mathcal{W}_{\beta}^{\delta}(\alpha, \gamma)$, the function $\mathcal{B}_{c}^{\delta}(f)(z) \in \mathcal{W}_{\xi}^{\delta}(\alpha, \gamma)$. The value of $\beta$ is sharp.

Let

$$
\lambda(t)=\frac{\Gamma(c)}{\Gamma(a) \Gamma(b) \Gamma(c-a-b+1)} t^{b-1}(1-t)^{c-a-b}{ }_{2} F_{1}\left(\begin{array}{c}
c-a, \quad 1-a \\
c-a-b+1
\end{array} ; 1-t\right),
$$

then the integral operator $(1)$ defined by the above weight function $\lambda(t)$ is the known as generalized Hohlov operator denoted by $\mathcal{H}_{a, b, c}^{\delta}$. This integral operator was considered in the work of A. Ebadian et al. [15]. Its representation in the form of convolution 
is given as $\mathcal{H}:=\mathcal{H}_{a, b, c}^{\delta}(f)(z)=\left(z^{\delta}{ }_{2} F_{1}(a, b ; c ; z)\right) *(f(z))^{\delta}$. For the case $\delta=1$, the convolution form of the reduced integral transform is given in the work of Y. C. Kim and F. Ronning [17] and studied by several authors later. The operator $\mathcal{H}_{a, b, c}^{\delta}$ with $a=1$ is the generalized Carlson-Shaffer operator $\left(\mathcal{L}_{b, c}^{\delta}\right)$ [8]. Corresponding to these operators the following results are obtained.

Theorem 2.3. Let $\alpha>\gamma\left(1+\frac{(2 a+1)}{\delta}\right) \geq 0, \delta>0,0<a \leq \min \left\{1, \frac{\delta}{2(\alpha-\gamma)}\right\}$ and $0<1+b<(c-a)<2$. Then for $f(z) \in \mathcal{W}_{\beta_{1}}^{\delta}(0,0)$, the function $\mathcal{H}_{a, b, c}^{\delta}(f)(z)$ belongs to the class $\mathcal{W}_{\beta}^{\delta}(\alpha, \gamma)$, where $\beta=1-2\left(1-\beta_{1}\right)\left(1-\beta_{2}\right)$ with

$$
\begin{array}{r}
\beta_{2}:=\left[1-\frac{a}{\delta}\left(\alpha-\gamma\left(1+\frac{a}{\delta}\right)\right)\right]{ }_{2} F_{1}(a, b ; c ;-1)+\frac{a}{\delta}\left[\alpha-\gamma\left(1+\frac{(2 a+1)}{\delta}\right)\right]{ }_{2} F_{1}(a+1, b ; c ;-1) \\
+\frac{a(a+1) \gamma}{\delta^{2}}{ }_{2} F_{1}(a+2, b ; c ;-1) .
\end{array}
$$

The value of $\beta$ is sharp.

For $a=1$, Theorem 2.3 lead to the following result.

Corollary 2.3. Let $\alpha>\gamma\left(1+\frac{3}{\delta}\right) \geq 0, \delta>0$, and $0<b<(c-1)<1$. Then for $f(z) \in \mathcal{W}_{\beta_{1}}^{\delta}(0,0)$, the function $\mathcal{L}_{b, c}^{\delta}(f)(z)$ belongs to the class $\mathcal{W}_{\beta}^{\delta}(\alpha, \gamma)$, where $\beta=1-2\left(1-\beta_{1}\right)\left(1-\beta_{2}\right)$ with

$$
\begin{array}{r}
\beta_{2}:=\left[1-\frac{1}{\delta}\left(\alpha-\gamma\left(1+\frac{1}{\delta}\right)\right)\right]{ }_{2} F_{1}(1, b ; c ;-1)+\frac{1}{\delta}\left[\alpha-\gamma\left(1+\frac{3}{\delta}\right)\right]{ }_{2} F_{1}(2, b ; c ;-1) \\
+\frac{2 \gamma}{\delta^{2}}{ }_{2} F_{1}(3, b ; c ;-1) .
\end{array}
$$

The value of $\beta$ is sharp.

It is important to note that similar results for various operators considered in $[12,13]$ by the authors of this work are already obtained and are appearing elsewhere.

\section{Proofs of theorems $2.1,2.2$ and 2.3}

Proof of Theorem 2.1. Since the case $\gamma=0(\mu=0, \nu=\alpha>0)$ corresponds to [1, Theorem 2.1], it is enough to obtain the condition for $\gamma>0$. Let

$$
H(z):=(1-\alpha+2 \gamma)\left(\frac{f}{z}\right)^{\delta}+\left(\alpha-3 \gamma+\gamma\left[\left(1-\frac{1}{\delta}\right)\left(\frac{z f^{\prime}}{f}\right)+\frac{1}{\delta}\left(1+\frac{z f^{\prime \prime}}{f^{\prime}}\right)\right]\right)\left(\frac{f}{z}\right)^{\delta}\left(\frac{z f^{\prime}}{f}\right) .
$$

Now, considering $P(z)=\left(\frac{f}{z}\right)^{\delta}$ a simple computation gives

$$
\left(1-\frac{1}{\delta}\right) P(z)+\left(\frac{1}{\delta}\right)(z P(z))^{\prime}=\left(\frac{f}{z}\right)^{\delta}\left(\frac{z f^{\prime}}{f}\right) .
$$


Now, differentiating (9) twice and applying successively in (8) leads to

$$
H(z)=\left(1-\frac{(\alpha-\gamma)}{\delta}+\frac{\gamma}{\delta^{2}}\right) P(z)+\left(\frac{(\alpha-\gamma)}{\delta}-\frac{2 \gamma}{\delta^{2}}\right)(z P(z))^{\prime}+\frac{\gamma}{\delta^{2}}\left(z(z P(z))^{\prime}\right)^{\prime}
$$

which upon using (3) leads to

$$
H(z)=\left(1-\frac{(\mu+\nu)}{\delta}+\frac{\mu \nu}{\delta^{2}}\right) P(z)+\left(\frac{(\mu+\nu)}{\delta}-\frac{2 \mu \nu}{\delta^{2}}\right)(z P(z))^{\prime}+\frac{\mu \nu}{\delta^{2}}\left(z(z P(z))^{\prime}\right)^{\prime} .
$$

Considering a series expansion of the form $P(z)=1+\sum_{n=1}^{\infty} b_{n} z^{n}$ in the above equality gives

$$
H(z)=1+\frac{1}{\delta^{2}} \sum_{n=1}^{\infty}(\delta+n \mu)(\delta+n \nu) b_{n} z^{n} .
$$

From (9) and (10), it is a simple exercise to see that

$$
\left(\frac{f}{z}\right)^{\delta}\left(\frac{z f^{\prime}}{f}\right)=\left(1+\delta \sum_{n=1}^{\infty} \frac{(n+\delta)}{(\delta+n \mu)(\delta+n \nu)} z^{n}\right) * H(z) .
$$

Since

$$
\begin{aligned}
1+\delta \sum_{n=1}^{\infty} \frac{(n+\delta)}{(\delta+n \mu)(\delta+n \nu)} z^{n}= & {\left[\left(\frac{1}{\delta}\right){ }_{3} F_{2}\left(2, \frac{\delta}{\nu}, \frac{\delta}{\mu} ;\left(1+\frac{\delta}{\nu}\right),\left(1+\frac{\delta}{\mu}\right) ; z\right)\right.} \\
& \left.+\left(1-\frac{1}{\delta}\right){ }_{3} F_{2}\left(1, \frac{\delta}{\nu}, \frac{\delta}{\mu} ;\left(1+\frac{\delta}{\nu}\right),\left(1+\frac{\delta}{\mu}\right) ; z\right)\right],
\end{aligned}
$$

(11) is equivalent to

$$
\begin{aligned}
\left(\frac{f}{z}\right)^{\delta}\left(\frac{z f^{\prime}}{f}\right)= & {\left[\left(\frac{1}{\delta}\right){ }_{3} F_{2}\left(2, \frac{\delta}{\nu}, \frac{\delta}{\mu} ;\left(1+\frac{\delta}{\nu}\right),\left(1+\frac{\delta}{\mu}\right) ; z\right)\right.} \\
& \left.+\left(1-\frac{1}{\delta}\right){ }_{3} F_{2}\left(1, \frac{\delta}{\nu}, \frac{\delta}{\mu} ;\left(1+\frac{\delta}{\nu}\right),\left(1+\frac{\delta}{\mu}\right) ; z\right)\right] * H(z) .
\end{aligned}
$$

Taking the logarithmic derivative on both sides of (1) we have

$$
\frac{z F_{\delta}^{\prime}(z)}{F_{\delta}(z)}\left(\frac{F_{\delta}(z)}{z}\right)^{\delta}=\int_{0}^{1} \frac{\lambda(t)}{1-t z} d t *\left(\left(\frac{z f^{\prime}(z)}{f(z)}\right)\left(\frac{f(z)}{z}\right)^{\delta}\right) .
$$

Now, substituting (12) in (13) will give

$$
\begin{aligned}
\frac{z F_{\delta}^{\prime}(z)}{F_{\delta}(z)}\left(\frac{F_{\delta}(z)}{z}\right)^{\delta}= & \int_{0}^{1} \frac{\lambda(t)}{1-t z} d t *\left[\left(\frac{1}{\delta}\right){ }_{3} F_{2}\left(2, \frac{\delta}{\nu}, \frac{\delta}{\mu} ;\left(1+\frac{\delta}{\nu}\right),\left(1+\frac{\delta}{\mu}\right) ; z\right)\right. \\
& \left.+\left(1-\frac{1}{\delta}\right){ }_{3} F_{2}\left(1, \frac{\delta}{\nu}, \frac{\delta}{\mu} ;\left(1+\frac{\delta}{\nu}\right),\left(1+\frac{\delta}{\mu}\right) ; z\right)\right] * H(z) .
\end{aligned}
$$


For $f(z) \in \mathcal{W}_{\beta}^{\delta}(\alpha, \gamma)$, then it is easy to see that for some $\phi \in \mathbb{R}, \operatorname{Re} e^{i \phi}(H(z)-\beta)>0$. Therefore, for $\gamma>0$, it is required to prove the claim that

$$
\begin{aligned}
\operatorname{Re} \int_{0}^{1} \lambda(t) & {\left[\left(\frac{1}{\delta}\right){ }_{3} F_{2}\left(2, \frac{\delta}{\nu}, \frac{\delta}{\mu} ;\left(1+\frac{\delta}{\nu}\right),\left(1+\frac{\delta}{\mu}\right) ; t z\right)\right.} \\
+ & \left.\left(1-\frac{1}{\delta}\right){ }_{3} F_{2}\left(1, \frac{\delta}{\nu}, \frac{\delta}{\mu} ;\left(1+\frac{\delta}{\nu}\right),\left(1+\frac{\delta}{\mu}\right) ; t z\right)\right] d t>1-\frac{1-\xi}{2(1-\beta)},
\end{aligned}
$$

which by applying Lemma 2.1, implies that $F_{\delta} \in \mathcal{W}_{\xi}^{\delta}(1,0)$. Now it is enough to verify inequality (14).

From the identity

$$
{ }_{3} F_{2}(2, a, b ; c, d ; z)=(c-1){ }_{3} F_{2}(1, a, b ; c-1, d ; z)-(c-2){ }_{3} F_{2}(1, a, b ; c, d ; z),
$$

it follows that

$$
\begin{array}{r}
{ }_{3} F_{2}\left(2, \frac{\delta}{\nu}, \frac{\delta}{\mu} ;\left(1+\frac{\delta}{\nu}\right),\left(1+\frac{\delta}{\mu}\right) ; z\right)=\left(\frac{\delta}{\nu}\right){ }_{2} F_{1}\left(1, \frac{\delta}{\mu} ;\left(1+\frac{\delta}{\mu}\right) ; z\right) \\
-\left(\frac{\delta}{\nu}-1\right){ }_{3} F_{2}\left(1, \frac{\delta}{\nu}, \frac{\delta}{\mu} ;\left(1+\frac{\delta}{\nu}\right),\left(1+\frac{\delta}{\mu}\right) ; z\right) .
\end{array}
$$

Therefore

$$
\begin{aligned}
& \operatorname{Re} \int_{0}^{1} \lambda(t)\left[\left(\frac{1}{\delta}\right){ }_{3} F_{2}\left(2, \frac{\delta}{\nu}, \frac{\delta}{\mu} ;\left(1+\frac{\delta}{\nu}\right),\left(1+\frac{\delta}{\mu}\right) ; t z\right)\right. \\
& \left.+\left(1-\frac{1}{\delta}\right){ }_{3} F_{2}\left(1, \frac{\delta}{\nu}, \frac{\delta}{\mu} ;\left(1+\frac{\delta}{\nu}\right),\left(1+\frac{\delta}{\mu}\right) ; t z\right)\right] d t \\
= & \operatorname{Re} \int_{0}^{1} \lambda(t)\left[\left(\frac{1}{\nu}\right){ }_{2} F_{1}\left(1, \frac{\delta}{\mu} ;\left(1+\frac{\delta}{\mu}\right) ; t z\right)+\left(1-\frac{1}{\nu}\right){ }_{3} F_{2}\left(1, \frac{\delta}{\nu}, \frac{\delta}{\mu} ;\left(1+\frac{\delta}{\nu}\right),\left(1+\frac{\delta}{\mu}\right) ; t z\right)\right] d t .
\end{aligned}
$$

Since the integral form of the following generalized hypergeometric function is given as

$$
{ }_{2} F_{1}\left(1, \frac{\delta}{\mu} ;\left(1+\frac{\delta}{\mu}\right) ; t z\right)=\int_{0}^{1} \frac{d s}{\left(1-t z s^{\mu / \delta}\right)}
$$

and

$$
{ }_{3} F_{2}\left(1, \frac{\delta}{\nu}, \frac{\delta}{\mu} ;\left(1+\frac{\delta}{\nu}\right),\left(1+\frac{\delta}{\mu}\right) ; t z\right)=\int_{0}^{1} \int_{0}^{1} \frac{d r d s}{\left(1-t z r^{\nu / \delta} s^{\mu / \delta}\right)},
$$

therefore

$$
\begin{aligned}
\operatorname{Re} \int_{0}^{1} \lambda(t)\left[\left(\frac{1}{\nu}\right){ }_{2}\right. & F_{1} \\
& \left.\left(1, \frac{\delta}{\mu} ;\left(1+\frac{\delta}{\mu}\right) ; t z\right)+\left(1-\frac{1}{\nu}\right){ }_{3} F_{2}\left(1, \frac{\delta}{\nu}, \frac{\delta}{\mu} ;\left(1+\frac{\delta}{\nu}\right),\left(1+\frac{\delta}{\mu}\right) ; t z\right)\right] d t \\
= & \operatorname{Re} \int_{0}^{1} \lambda(t)\left[\left(\frac{1}{\nu}\right) \int_{0}^{1} \frac{d s}{\left(1-t z s^{\mu / \delta}\right)}+\left(1-\frac{1}{\nu}\right) \int_{0}^{1} \int_{0}^{1} \frac{d r d s}{\left(1-t z r^{\nu / \delta} s^{\mu / \delta}\right)}\right] d t .
\end{aligned}
$$

It is evident that $\operatorname{Re}\left(\frac{1}{1-t z}\right)>\frac{1}{(1+t)}$, for $|z|<1$. Thus by the given hypothesis

$$
\operatorname{Re} \int_{0}^{1} \lambda(t)\left[\left(\frac{1}{\nu}\right) \int_{0}^{1} \frac{d s}{\left(1-t z s^{\mu / \delta}\right)}+\left(1-\frac{1}{\nu}\right) \int_{0}^{1} \int_{0}^{1} \frac{d r d s}{\left(1-t z r^{\nu / \delta} s^{\mu / \delta}\right)}\right] d t
$$




$$
\begin{aligned}
& \geq \int_{0}^{1} \lambda(t)\left[\left(\frac{1}{\nu}\right) \int_{0}^{1} \frac{d s}{\left(1+t s^{\mu / \delta}\right)}+\left(1-\frac{1}{\nu}\right) \int_{0}^{1} \int_{0}^{1} \frac{d r d s}{\left(1+t r^{\nu / \delta} s^{\mu / \delta}\right)}\right] d t \\
& =1-\frac{1-\xi}{2(1-\beta)}
\end{aligned}
$$

Here $\beta<1$ if and only if

$$
1-\frac{1-\xi}{2}\left(1-\int_{0}^{1} \lambda(t)\left[\frac{1}{\nu} \int_{0}^{1} \frac{d s}{1+t s^{\mu / \delta}}+\left(1-\frac{1}{\nu}\right) \int_{0}^{1} \int_{0}^{1} \frac{d r d s}{1+t r^{\nu / \delta} s^{\mu / \delta}}\right] d t\right)^{-1}<1
$$

Since $\xi<1$ and $\int_{0}^{1} \lambda(t) d t=1$, therefore the above condition holds if and only if

$$
\int_{0}^{1} \lambda(t)\left[1-\frac{1}{\nu} \int_{0}^{1} \frac{d s}{1+t s^{\mu / \delta}}-\left(1-\frac{1}{\nu}\right) \int_{0}^{1} \int_{0}^{1} \frac{d r d s}{1+t r^{\nu / \delta} s^{\mu / \delta}}\right] d t>0
$$

or $\int_{0}^{1} \lambda(t) G(t) d t>0$, where

$$
G(t)=\left[1-\frac{\delta}{\nu} \sum_{n=0}^{\infty} \frac{(-1)^{n} t^{n}}{(\delta+n \mu)}-\delta^{2}\left(1-\frac{1}{\nu}\right) \sum_{n=0}^{\infty} \frac{(-1)^{n} t^{n}}{(\delta+n \mu)(\delta+n \nu)}\right] .
$$

Since $\mu, \nu$ and $\delta$ are positive and $0<t<1$, therefore by Leibnitz's test both the series

$$
\sum_{n=0}^{\infty} \frac{(-1)^{n} t^{n}}{(\delta+n \mu)} \quad \text { and } \quad \sum_{n=0}^{\infty} \frac{(-1)^{n} t^{n}}{(\delta+n \mu)(\delta+n \nu)}
$$

are convergent with $\sum_{n=0}^{\infty} \frac{(-1)^{n} t^{n}}{(\delta+n \mu)}<\frac{1}{\delta}$ and $\sum_{n=0}^{\infty} \frac{(-1)^{n} t^{n}}{(\delta+n \mu)(\delta+n \nu)}<\frac{1}{\delta^{2}}$.

Further, using the previous conditions, it is easy to see that

$$
G(t)>\left[1-\frac{\delta}{\nu}\left(\frac{1}{\delta}\right)-\delta^{2}\left(1-\frac{1}{\nu}\right)\left(\frac{1}{\delta^{2}}\right)\right]=0 \quad \forall \quad t \in(0,1) .
$$

By the given hypothesis $\lambda(t)$ is a non-negative integrable function. Hence $\int_{0}^{1} \lambda(t) G(t) d t>$ 0 or $\beta<1$. This completes the proof.

Now, to verify the sharpness let $f(z) \in \mathcal{W}_{\beta}^{\delta}(\alpha, \gamma)$, therefore it satisfies the differential equation

$$
\frac{\mu \nu}{\delta^{2}} z^{1-\delta / \mu}\left(z^{\delta / \mu-\delta / \nu+1}\left(z^{\delta / \nu}\left(\frac{f}{z}\right)^{\delta}\right)^{\prime}\right)^{\prime}=\beta+(1-\beta) \frac{1+z}{1-z}
$$

with $\beta<1$ defined in (4). From (3), (15) and the series representation of $P(z)$, an easy calculation gives

$$
\left(\frac{f(z)}{z}\right)^{\delta}\left(\frac{z f^{\prime}(z)}{f(z)}\right)=1+2(1-\beta) \sum_{n=1}^{\infty} \frac{\delta(n+\delta) z^{n}}{(\delta+n \nu)(\delta+n \mu)} .
$$


Therefore from (13), we have

$$
\begin{aligned}
\left(\frac{F_{\delta}(z)}{z}\right)^{\delta}\left(\frac{z F_{\delta}^{\prime}(z)}{F_{\delta}(z)}\right) & =\int_{0}^{1} \lambda(t)\left(1+2(1-\beta) \sum_{n=1}^{\infty} \frac{\delta(n+\delta)(t z)^{n}}{(\delta+n \nu)(\delta+n \mu)}\right) d t \\
& =1+2(1-\beta) \sum_{n=1}^{\infty} \frac{\delta(n+\delta) \tau_{n} z^{n}}{(\delta+n \nu)(\delta+n \mu)}
\end{aligned}
$$

where $\tau_{n}=\int_{0}^{1} t^{n} \lambda(t) d t$. By the simple adjustment, (4) can be written as

$$
\frac{1}{(1-\beta)}=\frac{2}{(1-\xi)}\left(1-\int_{0}^{1} \lambda(t)\left[\frac{1}{\nu} \int_{0}^{1} \frac{d s}{1+t s^{\mu / \delta}}+\left(1-\frac{1}{\nu}\right) \int_{0}^{1} \int_{0}^{1} \frac{d r d s}{1+t r^{\nu / \delta} s^{\mu / \delta}}\right] d t\right)
$$

or equivalently,

$$
\begin{aligned}
& \frac{1}{(1-\beta)}=\frac{2}{(1-\xi)}\left(1-\int_{0}^{1} \lambda(t)\left[\frac{\delta}{\nu} \sum_{n=0}^{\infty} \frac{(-1)^{n} t^{n}}{(\delta+n \mu)}+\delta^{2}\left(1-\frac{1}{\nu}\right) \sum_{n=0}^{\infty} \frac{(-1)^{n} t^{n}}{(\delta+n \mu)(\delta+n \nu)}\right] d t\right) . \\
& \text { As } \tau_{n}=\int_{0}^{1} t^{n} \lambda(t) d t \\
& \qquad \frac{1}{(1-\beta)}=\frac{2}{(1-\xi)}\left(1-\frac{\delta}{\nu} \sum_{n=0}^{\infty} \frac{(-1)^{n} \tau_{n}}{(\delta+n \mu)}-\delta^{2}\left(1-\frac{1}{\nu}\right) \sum_{n=0}^{\infty} \frac{(-1)^{n} \tau_{n}}{(\delta+n \mu)(\delta+n \nu)}\right)
\end{aligned}
$$

or

$$
\xi=1+2(1-\beta) \sum_{n=1}^{\infty} \frac{(-1)^{n} \delta(n+\delta) \tau_{n}}{(\delta+n \mu)(\delta+n \nu)} .
$$

Further using (16) and (17), we have

$$
\left.\left(\frac{F_{\delta}(z)}{z}\right)^{\delta}\left(\frac{z F_{\delta}^{\prime}(z)}{F_{\delta}(z)}\right)\right|_{z=-1}=1+2(1-\beta) \sum_{n=1}^{\infty} \frac{(-1)^{n} \delta(n+\delta) \tau_{n}}{(\delta+n \mu)(\delta+n \nu)}=\xi,
$$

which clearly implies the sharpness of the result.

Proof of Theorem 2.2. Since the case $\gamma=0(\mu=0, \nu=\alpha>0)$ corresponds to $[1$, Theorem 2.2], therefore the case $\gamma>0$ is taken into consideration. Now, for the function $f(z) \in \mathcal{W}_{\beta}^{\delta}(\alpha, \gamma)$, let

$$
G(z)=\frac{H(z)-\beta}{(1-\beta)} .
$$

Then it is easy to see that for some $\phi \in \mathbb{R}, \operatorname{Re}\left(e^{i \phi} G(z)\right)>0$, where $H(z)$ is defined in (8).

Now, the following two cases are discussed.

At first, let $\gamma \neq \delta \alpha /(3 \delta-1)$. From (8), (13) and (18),

$$
\left(\frac{F_{\delta}(z)}{z}\right)^{\delta}\left(\frac{z F_{\delta}^{\prime}(z)}{F_{\delta}(z)}\right)=\int_{0}^{1} \frac{\lambda(t)}{(1-t z)} d t * \frac{1}{(\alpha-3 \gamma+\gamma / \delta)}\left(\beta+(1-\beta) G(z)-(1-\alpha+2 \gamma)\left(\frac{f}{z}\right)^{\delta}\right.
$$




$$
\left.-\gamma\left(\left(1-\frac{1}{\delta}\right)\left(\frac{z f^{\prime}}{f}\right)+\frac{1}{\delta}\left(\frac{z f^{\prime \prime}}{f^{\prime}}\right)\right)\left(\frac{f}{z}\right)^{\delta}\left(\frac{z f^{\prime}}{f}\right)\right) .
$$

Now, from (1) and (13), it is a simple exercise to see that

$$
\begin{aligned}
& \left(\left(1-\frac{1}{\delta}\right)\left(\frac{z F_{\delta}^{\prime}(z)}{F_{\delta}(z)}\right)+\frac{1}{\delta}\left(\frac{z F_{\delta}^{\prime \prime}(z)}{F_{\delta}^{\prime}(z)}\right)\right)\left(\frac{F_{\delta}(z)}{z}\right)^{\delta}\left(\frac{z F_{\delta}^{\prime}(z)}{F_{\delta}(z)}\right) \\
& =\int_{0}^{1} \frac{\lambda(t)}{1-t z} d t *\left(\left(1-\frac{1}{\delta}\right)\left(\frac{z f^{\prime}(z)}{f(z)}\right)+\frac{1}{\delta}\left(\frac{z f^{\prime \prime}(z)}{f^{\prime}(z)}\right)\right)\left(\frac{f(z)}{z}\right)^{\delta}\left(\frac{z f^{\prime}(z)}{f(z)}\right) .
\end{aligned}
$$

Therefore (19) and (20) leads to

$$
\begin{aligned}
\left(\frac{F_{\delta}(z)}{z}\right)^{\delta}\left(\frac{z F_{\delta}^{\prime}(z)}{F_{\delta}(z)}\right)= & {\left[\left(\beta+(1-\beta) \int_{0}^{1} \frac{\lambda(t)}{(1-t z)} d t\right) * \frac{1}{(\alpha-3 \gamma+\gamma / \delta)} G(z)\right] } \\
& -\frac{1}{(\alpha-3 \gamma+\gamma / \delta)}\left((1-\alpha+2 \gamma)\left(\frac{F_{\delta}(z)}{z}\right)^{\delta}+\gamma\left(\left(1-\frac{1}{\delta}\right)\left(\frac{z F_{\delta}^{\prime}(z)}{F_{\delta}(z)}\right)\right.\right. \\
& \left.\left.+\frac{1}{\delta}\left(\frac{z F_{\delta}^{\prime \prime}(z)}{F_{\delta}^{\prime}(z)}\right)\right)\left(\frac{F_{\delta}(z)}{z}\right)^{\delta}\left(\frac{z F_{\delta}^{\prime}(z)}{F_{\delta}(z)}\right)\right)
\end{aligned}
$$

or equivalently,

$$
\begin{aligned}
(\beta+ & \left.(1-\beta) \int_{0}^{1} \frac{\lambda(t)}{(1-t z)} d t\right) * G(z)=(1-\alpha+2 \gamma)\left(\frac{F_{\delta}(z)}{z}\right)^{\delta} \\
& +\left(\alpha-3 \gamma+\gamma\left[\left(1-\frac{1}{\delta}\right)\left(\frac{z F_{\delta}^{\prime}(z)}{F_{\delta}(z)}\right)+\frac{1}{\delta}\left(1+\frac{z F_{\delta}^{\prime \prime}(z)}{F_{\delta}^{\prime}(z)}\right)\right]\right)\left(\frac{F_{\delta}(z)}{z}\right)^{\delta}\left(\frac{z F_{\delta}^{\prime}(z)}{F_{\delta}(z)}\right) .
\end{aligned}
$$

Now consider $\gamma=\delta \alpha /(3 \delta-1)$. It is easy to see that the equation (18) is equivalent to

$$
\begin{array}{r}
\left(1-\gamma+\frac{\gamma}{\delta}\right)\left(\frac{f}{z}\right)^{\delta}+\gamma\left(\left(1-\frac{1}{\delta}\right)\left(\frac{z f^{\prime}}{f}\right)+\frac{1}{\delta}\left(\frac{z f^{\prime \prime}}{f^{\prime}}\right)\right)\left(\frac{f}{z}\right)^{\delta}\left(\frac{z f^{\prime}}{f}\right) \\
=\beta+(1-\beta) G(z) .
\end{array}
$$

Therefore the above expression along with (13) and (20) gives

$$
\begin{aligned}
G(z) *(\beta & \left.+(1-\beta) \int_{0}^{1} \frac{\lambda(t)}{(1-t z)} d t\right)=\left(1-\gamma+\frac{\gamma}{\delta}\right)\left(\frac{F_{\delta}(z)}{z}\right)^{\delta} \\
& +\gamma\left(\left(1-\frac{1}{\delta}\right)\left(\frac{z F_{\delta}^{\prime}(z)}{F_{\delta}(z)}\right)+\frac{1}{\delta}\left(\frac{z F_{\delta}^{\prime \prime}(z)}{F_{\delta}^{\prime}(z)}\right)\right)\left(\frac{F_{\delta}(z)}{z}\right)^{\delta}\left(\frac{z F_{\delta}^{\prime}(z)}{F_{\delta}(z)}\right),
\end{aligned}
$$

which coincides with $(21)$, when $\gamma=\delta \alpha /(3 \delta-1)$.

Moreover, $F_{\delta}(z) \in \mathcal{W}_{\xi}^{\delta}(\alpha, \gamma)$ if, and only if, $J(z) \in \mathcal{W}_{0}^{\delta}(\alpha, \gamma)$ where the functions $F_{\delta}(z)$ and $J(z)$ are defined by the relation

$$
\left(\frac{J(z)}{z}\right)^{\delta}=\frac{\left(\frac{F_{\delta}(z)}{z}\right)^{\delta}-\xi}{(1-\xi)} \Longleftrightarrow\left(\frac{F_{\delta}(z)}{z}\right)^{\delta}=(1-\xi)\left(\frac{J(z)}{z}\right)^{\delta}+\xi .
$$


From the above expression, a simple computation give

$$
\left(\frac{F_{\delta}(z)}{z}\right)^{\delta}\left(\frac{z F_{\delta}^{\prime}(z)}{F_{\delta}(z)}\right)=(1-\xi)\left(\frac{J(z)}{z}\right)^{\delta}\left(\frac{z J^{\prime}(z)}{J(z)}\right)+\xi .
$$

Further using (23) will give

$$
\begin{aligned}
& \left(\left(1-\frac{1}{\delta}\right)\left(\frac{z F_{\delta}^{\prime}(z)}{F_{\delta}(z)}\right)+\frac{1}{\delta}\left(\frac{z F_{\delta}^{\prime \prime}(z)}{F_{\delta}^{\prime}(z)}\right)\right)\left(\frac{F_{\delta}(z)}{z}\right)^{\delta}\left(\frac{z F_{\delta}^{\prime}(z)}{F_{\delta}(z)}\right) \\
& =(1-\xi)\left(\left(1-\frac{1}{\delta}\right)\left(\frac{z J^{\prime}(z)}{J(z)}\right)+\frac{1}{\delta}\left(\frac{z J^{\prime \prime}(z)}{J^{\prime}(z)}\right)\right)\left(\frac{J(z)}{z}\right)^{\delta}\left(\frac{z J^{\prime}(z)}{J(z)}\right)+\xi(1-\delta) .
\end{aligned}
$$

Putting the values from (22), (23) and (24) in (18), it follows that

$$
\begin{aligned}
G(z) & =(1-\alpha+2 \gamma)\left(\frac{J(z)}{z}\right)^{\delta} \\
& +\left(\alpha-3 \gamma+\gamma\left[\left(1-\frac{1}{\delta}\right)\left(\frac{z J^{\prime}(z)}{J(z)}\right)+\frac{1}{\delta}\left(1+\frac{z J^{\prime \prime}(z)}{J^{\prime}(z)}\right)\right]\right)\left(\frac{J(z)}{z}\right)^{\delta}\left(\frac{z J^{\prime}(z)}{J(z)}\right),
\end{aligned}
$$

which implies that the function $J(z) \in \mathcal{W}_{0}^{\delta}(\alpha, \gamma)$. Further using (21) will give

$$
\begin{aligned}
\left(\frac{\beta-\xi}{1-\xi}+\right. & \left.\frac{1-\beta}{1-\xi} \int_{0}^{1} \frac{\lambda(t)}{(1-t z)} d t\right) * G(z)=(1-\alpha+2 \gamma)\left(\frac{J(z)}{z}\right)^{\delta} \\
& +\left(\alpha-3 \gamma+\gamma\left[\left(1-\frac{1}{\delta}\right)\left(\frac{z J^{\prime}(z)}{J(z)}\right)+\frac{1}{\delta}\left(1+\frac{z J^{\prime \prime}(z)}{J^{\prime}(z)}\right)\right]\right)\left(\frac{J(z)}{z}\right)^{\delta}\left(\frac{z J^{\prime}(z)}{J(z)}\right) .
\end{aligned}
$$

As $\operatorname{Re}\left(e^{i \phi} G(z)\right)>0$, from the result given in [21, P. 23] and by the above expression, it is easy to note that

$(1-\alpha+2 \gamma)\left(\frac{J(z)}{z}\right)^{\delta}+\left(\alpha-3 \gamma+\gamma\left[\left(1-\frac{1}{\delta}\right)\left(\frac{z J^{\prime}(z)}{J(z)}\right)+\frac{1}{\delta}\left(1+\frac{z J^{\prime \prime}(z)}{J^{\prime}(z)}\right)\right]\right)\left(\frac{J(z)}{z}\right)^{\delta}\left(\frac{z J^{\prime}(z)}{J(z)}\right) \neq 0$,

if, and only if,

$$
\operatorname{Re}\left(\frac{\beta-\xi}{1-\xi}+\frac{1-\beta}{1-\xi} \int_{0}^{1} \frac{\lambda(t)}{(1-t z)} d t\right)>\frac{1}{2} .
$$

Now, using $\operatorname{Re}\left(\frac{1}{1-t z}\right)>\left(\frac{1}{1+t}\right),|z|<1$, in the above inequality, it follows that

$$
\operatorname{Re}\left(\frac{\beta-\xi}{1-\xi}+\frac{1-\beta}{1-\xi} \int_{0}^{1} \frac{\lambda(t)}{(1-t z)} d t\right)>\frac{\beta-\xi}{1-\xi}+\frac{1-\beta}{1-\xi} \int_{0}^{1} \frac{\lambda(t)}{(1+t)} d t .
$$

Since the expression (7) can be rewritten as

$$
\frac{\beta-\xi}{1-\beta}+\int_{0}^{1} \frac{\lambda(t)}{(1+t)} d t=\frac{(1-\xi)}{2(1-\beta)},
$$

the above inequality gives

$$
\operatorname{Re}\left(\frac{\beta-\xi}{1-\xi}+\frac{1-\beta}{1-\xi} \int_{0}^{1} \frac{\lambda(t)}{(1-t z)} d t\right)>\frac{\beta-\xi}{1-\xi}+\frac{1-\beta}{1-\xi} \int_{0}^{1} \frac{\lambda(t)}{(1+t)} d t=\frac{1}{2}
$$

and this completes the proof. 
Now, to verify the sharpness let $f(z) \in \mathcal{W}_{\beta}^{\delta}(\alpha, \gamma)$. Using the series representation of $f(z)$ in (1) will give

$$
\left(\frac{F_{\delta}(z)}{z}\right)^{\delta}=1+2(1-\beta) \sum_{n=1}^{\infty} \frac{\delta^{2} \tau_{n} z^{n}}{(\delta+n \nu)(\delta+n \mu)}
$$

where $\tau_{n}=\int_{0}^{1} t^{n} \lambda(t) d t$. Now, consider

$$
\begin{aligned}
H_{0}(z) & :=(1-\alpha+2 \gamma)\left(\frac{F_{\delta}(z)}{z}\right)^{\delta} \\
& +\left(\alpha-3 \gamma+\gamma\left[\left(1-\frac{1}{\delta}\right)\left(\frac{z F_{\delta}^{\prime}(z)}{F_{\delta}(z)}\right)+\frac{1}{\delta}\left(1+\frac{z F_{\delta}^{\prime \prime}(z)}{F_{\delta}^{\prime}(z)}\right)\right]\right)\left(\frac{F_{\delta}(z)}{z}\right)^{\delta}\left(\frac{z F_{\delta}^{\prime}(z)}{F_{\delta}(z)}\right)
\end{aligned}
$$

Therefore, from (25), a simple calculation gives

$$
H_{0}(z)=1+2(1-\beta) \sum_{n=1}^{\infty} \tau_{n} z^{n}
$$

Now rewriting (7) as

$$
\frac{1}{1-\beta}=-\frac{2}{(1-\xi)} \sum_{n=1}^{\infty}(-1)^{n} \tau_{n} .
$$

and finding $H_{0}(z)$ at $z=-1$ gives $H_{0}(-1)=\xi$, which clearly implies the sharpness of the result.

Proof of Theorem 2.3. Since $\mathcal{H}^{\delta}=z^{\delta}{ }_{2} F_{1}(a, b ; c ; z) * f(z)$, it is a simple exercise to note that

$$
\left(\frac{\mathcal{H}}{z}\right)^{\delta}\left(\frac{z \mathcal{H}^{\prime}}{\mathcal{H}}\right)=N_{1}(z) *\left(\frac{f(z)}{z}\right)^{\delta}
$$

where $N_{1}(z):={ }_{2} F_{1}(a, b ; c ; z)+\frac{a b}{c \delta} z{ }_{2} F_{1}(a+1, b+1 ; c+1 ; z)$. Further from the above expression, it follows that

$$
\left[\left(1-\frac{1}{\delta}\right)\left(\frac{z \mathcal{H}^{\prime}}{\mathcal{H}}\right)+\frac{1}{\delta}\left(\frac{z \mathcal{H}^{\prime \prime}}{\mathcal{H}^{\prime}}\right)\right]\left(\frac{\mathcal{H}}{z}\right)^{\delta}\left(\frac{z \mathcal{H}^{\prime}}{\mathcal{H}}\right)=N_{2}(z) *\left(\frac{f(z)}{z}\right)^{\delta}
$$

where

$$
\begin{array}{r}
N_{2}(z):=\left(1-\frac{1}{\delta}\right) \\
{ }_{2} F_{1}(a, b ; c ; z)+\frac{2 a b}{c \delta} z{ }_{2} F_{1}(a+1, b+1 ; c+1 ; z) \\
+\frac{a(a+1) b(b+1)}{c(c+1) \delta^{2}} z^{2}{ }_{2} F_{1}(a+2, b+2 ; c+2 ; z) .
\end{array}
$$

Therefore

$$
\begin{array}{r}
\left(\alpha-3 \gamma+\gamma\left[\left(1-\frac{1}{\delta}\right)\right.\right. \\
\left.\left.\left(\frac{z f^{\prime}}{f}\right)+\frac{1}{\delta}\left(1+\frac{z f^{\prime \prime}}{f^{\prime}}\right)\right]\right)\left(\frac{f}{z}\right)^{\delta}\left(\frac{z f^{\prime}}{f}\right) \\
+(1-\alpha+2 \gamma)\left(\frac{f}{z}\right)^{\delta}=N_{3}(z) *\left(\frac{f(z)}{z}\right)^{\delta}
\end{array}
$$


where

$$
\begin{array}{r}
N_{3}(z):={ }_{2} F_{1}(a, b ; c ; z)+\frac{a b}{c \delta}\left(\alpha-\gamma+\frac{\gamma}{\delta}\right) z_{2} F_{1}(a+1, b+1 ; c+1 ; z) \\
+\frac{a(a+1) b(b+1) \gamma}{c(c+1) \delta^{2}} z^{2}{ }_{2} F_{1}(a+2, b+2 ; c+2 ; z) .
\end{array}
$$

From the contiguous relation for Gaussian hypergeometric functions [5, Page 96] (or the verification can be made by comparing the coefficient of $z^{n}$ both the sides)

$$
b_{2} F_{1}(a+1, b+1 ; c+1 ; z)=c\left({ }_{2} F_{1}(a+1, b ; c ; z)-{ }_{2} F_{1}(a, b ; c ; z)\right),
$$

it follows that

$$
\begin{array}{r}
N_{3}(z)=\left[1-\frac{a}{\delta}\left(\alpha-\gamma\left(1+\frac{a}{\delta}\right)\right)\right]{ }_{2} F_{1}(a, b ; c ; z)+\frac{a}{\delta}\left[\alpha-\gamma\left(1+\frac{(2 a+1)}{\delta}\right)\right]{ }_{2} F_{1}(a+1, b ; c ; z) \\
+\frac{a(a+1) \gamma}{\delta^{2}}{ }_{2} F_{1}(a+2, b ; c ; z) .
\end{array}
$$

For $a>0, b>0$ and $c>0$, the integral representation of Gaussian hypergeometric function is given as [17]

$$
{ }_{2} F_{1}(a, b ; c ; z)=\frac{\Gamma(c)}{\Gamma(a) \Gamma(b) \Gamma(c-a-b+1)} \int_{0}^{1} t^{b-1}(1-t)^{c-a-b}{ }_{2} F_{1}\left(\begin{array}{c}
c-a, 1-a \\
c-a-b+1
\end{array} ; 1-t\right) \frac{1}{(1-t z)} d t .
$$

In view of the above integral form, it is easy to see that

$$
N_{3}(z)=\frac{\Gamma(c)}{\Gamma(a) \Gamma(b) \Gamma(c-a-b-1)} \int_{0}^{1} t^{b-1}(1-t)^{c-a-b-2} \frac{N_{4}(t)}{(1-t z)} d t,
$$

where

$$
\begin{aligned}
N_{4}(t):= & {\left[1-\frac{a}{\delta}\left(\alpha-\gamma\left(1+\frac{a}{\delta}\right)\right)\right](1-t)^{2} \frac{{ }_{2} F_{1}(c-a, 1-a ; c-a-b+1 ; 1-t)}{(c-a-b)(c-a-b-1)} } \\
& +\frac{a}{\delta}\left[\alpha-\gamma\left(1+\frac{(2 a+1)}{\delta}\right)\right](1-t) \frac{{ }_{2} F_{1}(c-a-1,-a ; c-a-b ; 1-t)}{a(c-a-b-1)} \\
& +\frac{\gamma}{\delta^{2}}{ }_{2} F_{1}(c-a-2,-(a+1) ; c-a-b-1 ; 1-t) \\
= & e_{1}+e_{2}(1-t)+\sum_{n=0}^{\infty} e_{3} \frac{(c-a)_{n}(1-a)_{n}}{(c-a-b)_{n+1}(3)_{n}}(1-t)^{n+2},
\end{aligned}
$$

where

$$
\begin{aligned}
e_{1}:= & \frac{\gamma}{\delta^{2}} \\
e_{2}:= & \frac{1}{\delta^{2}(c-a-b-1)}(\alpha \delta-\gamma(\delta+2 a+1)-\gamma(a+1)(c-a-2)) \\
e_{3}:= & \frac{1}{2 \delta^{2}(c-a-b)(c-a-b-1)}\left(n^{2}\left(\delta^{2}-a \alpha \delta+a \gamma \delta+a^{2} \gamma\right)\right. \\
& +n\left[3\left(\delta^{2}-a \alpha \delta+a \gamma \delta+a^{2} \gamma\right)-a(\alpha \delta-\gamma(\delta+2 a+1))(c-a-1)\right]
\end{aligned}
$$




$$
\begin{aligned}
& +2\left(\delta^{2}-a \alpha \delta+a \gamma \delta+a^{2} \gamma\right)-a(c-a-1)[2(\alpha \delta-\gamma(\delta+2 a+1)) \\
& -\gamma(a+1)(c-a-2)])
\end{aligned}
$$

which is non-negative when $0<1+b<(c-a)<2,0<a \leq \min \left\{1, \frac{\delta}{2(\alpha-\gamma)}\right\}$ and $\alpha>\gamma\left(1+\frac{(2 a+1)}{\delta}\right) \geq 0$. Since $\operatorname{Re}\left(\frac{1}{1-t z}\right)>\frac{1}{(1+t)}$, for $|z|<1$, which on using $N_{3}(z)$ gives $\operatorname{Re} N_{3}(z)>N_{3}(-1)$. Applying Lemma 2.1 will give the required result.

Now, to verify the sharpness, consider the function

$$
\left(\frac{f(z)}{z}\right)^{\delta}=\beta_{1}+\left(1-\beta_{1}\right) \frac{1+z}{1-z} \quad \text { and } \quad N_{3}(z)=\beta_{2}+\left(1-\beta_{2}\right) \frac{1+z}{1-z} .
$$

Since $\beta=1-2\left(1-\beta_{1}\right)\left(1-\beta_{2}\right)$, therefore

$$
\left(\frac{f(z)}{z}\right)^{\delta} * N_{3}(z)=1+4\left(1-\beta_{1}\right)\left(1-\beta_{2}\right) \frac{1+z}{1-z}=\beta+(1-\beta) \frac{1+z}{1-z}
$$

which clearly implies the sharpness of the result.

\section{References}

[1] R. Aghalary, A. Ebadian and S. Shams, Geometric properties of some linear operators defined by convolution, Tamkang J. Math. 39 (2008), no. 4, 325-334.

[2] R. M. Ali, A. O. Badghaish, V. Ravichandran and A. Swaminathan, Starlikeness of integral transforms and duality, J. Math. Anal. Appl. 385 (2012), no. 2, 808-822.

[3] R. M. Ali, M. M. Nargesi and V. Ravichandran, Convexity of integral transforms and duality, Complex Var. Elliptic Equ. 58 (2013), no. 11, 1569-1590.

[4] R. M. Ali and V. Singh, Convexity and starlikeness of functions defined by a class of integral operators, Complex Variables Theory Appl. 26 (1995), no. 4, 299-309.

[5] G. E. Andrews, R. Askey and R. Roy, Special functions, Encyclopedia of Mathematics and its Applications, 71, Cambridge Univ. Press, Cambridge, 1999.

[6] T. Bulboacă, Differential Subordinations and Superordinations. Recent Results, House of Scientific Book Publications, Cluj-Napoca, 2005.

[7] S. D. Bernardi, Convex and starlike univalent functions, Trans. Amer. Math. Soc. 135 (1969), 429-446.

[8] B. C. Carlson and D. B. Shaffer, Starlike and prestarlike hypergeometric functions, SIAM J. Math. Anal. 15 (1984), no. 4, 737-745.

[9] J. H. Choi, Y. C. Kim and M. Saigo, Geometric properties of convolution operators defined by Gaussian hypergeometric functions, Integral Transforms Spec. Funct. 13 (2002), no. 2, 117-130.

[10] S. Devi and A. Swaminathan, Integral transforms of functions to be in a class of analytic functions using duality techniques, J. Complex Anal. 2014, Art. ID 473069, 10 pp.

[11] S. Devi and A. Swaminathan, Order of Starlikeness and Convexity of certain integral transforms using duality techniques, 16 pages, Submitted for publication, (http://arxiv.org/abs/1406.6471).

[12] S. Devi and A. Swaminathan, Starlikeness of the Generalized Integral Transform using Duality Techniques, 24 pages, Submitted for publication, (http://arxiv.org/abs/1411.5217).

[13] S. Devi and A. Swaminathan, Convexity of the Generalized Integral Transform using Duality Techniques, Submitted for publication. (http://arxiv.org/abs/1411.5898)

[14] S. S. Ding, Y. Ling and G. J. Bao, Some properties of a class of analytic functions, J. Math. Anal. Appl. 195 (1995), no. 1, 71-81. 
[15] A. Ebadian, R. Aghalary and S. Shams, Application of duality techniques to starlikeness of weighted integral transforms, Bull. Belg. Math. Soc. Simon Stevin 17 (2010), no. 2, 275-285.

[16] R. Fournier and S. Ruscheweyh, On two extremal problems related to univalent functions, Rocky Mountain J. Math. 24 (1994), no. 2, 529-538.

[17] Y. C. Kim and F. Rønning, Integral transforms of certain subclasses of analytic functions, J. Math. Anal. Appl. 258 (2001), no. 2, 466-489.

[18] M. Liu, Properties for some subclasses of analytic functions, Bull. Inst. Math. Acad. Sinica 30 (2002), no. 1, 9-26.

[19] S. S. Miller and P. T. Mocanu, Differential Subordinations, Dekker, New York, 2000.

[20] S. S. Miller and P. T. Mocanu, A class of nonlinear averaging integral operators, J. Math. Anal. Appl. 197 (1996), no. 1, 318-323.

[21] S. Ruscheweyh, Convolutions in geometric function theory, Séminaire de Mathématiques Supérieures, 83, Presses Univ. Montréal, Montreal, QC, 1982.

[22] J. Stankiewicz and Z. Stankiewicz, Some applications of the Hadamard convolution in the theory of functions, Ann. Univ. Mariae Curie-Skłodowska Sect. A 40 (1986), 251-265 (1987).

[23] S. Verma, S. Gupta and S. Singh, On an integral transform of a class of analytic functions, Abstr. Appl. Anal. 2012, Art. ID 259054, 10 pp. 